Rev. Col. Ciencia. Vol. 2, no. 1. Octubre 2020 - marzo 2021. ISSN L 2710-7434 pp. 58-71

\title{
DESCRIPCIÓN DE LOS PRINCIPALES SITOS DE BUCEO EN LA COSTA DE PIXVAE, PACÍFICO PANAMEÑO
}

\section{DESCRIPTION OF THE MAIN DIVING SITES IN THE PIXVAE COAST FROM PANAMANIAN PACIFIC}

\author{
Luis A. Montes ${ }^{1}$, Ángel J. Vega ${ }^{12}$, Kevan Mantell ${ }^{3}$ y Yolani A. Robles P. ${ }^{1}$ \\ ${ }^{1}$ Universidad de Panamá - Centro de Capacitación, Investigación y Monitoreo de la Biodiversidad en el \\ Parque Nacional Coiba (CCIMBIO-COIBA-UP); montes lu@hotmail.com; angel.vega@up.ac.pa; \\ yolany.robles@up.ac.pa Panamá \\ 2 Estación Científica del Parque Nacional Coiba (Coiba-AIP) \\ 3Dive Base Coiba; uwkevan@gmail.com
}

\begin{abstract}
RESUMEN
Los ambientes marinos en la franja continental del área de influencia del Parque Nacional Coiba (PNC), cuenta con importantes atributos ecológicos que mantienen una estrecha relación con el área protegida. Es así que, con la finalidad de caracterizar los fondos marinos en la ensenada de Pixvae, área de co-manejo para la pesca responsable, se realizaron descripciones de los principales atributos donde se documenta una serie de componentes ecológicos por medio de fotografías y bosquejos de los fondos, para representar las características ecológicas del sitio y la diversidad marina que alberga, y brinda la oportunidad para desarrollar actividades sustentables independientes a la pesca, como el buceo recreativo y científico, lo que puede repercutir en alternativas económicas para la comunidad, enfocadas en la recuperación y conservación del sistema. Sin duda, las características de las comunidades coralinas asociadas a los fondos de la ensenada de Pixvae presentan atributos que las hacen atractivas para desarrollar el turismo ecológico.
\end{abstract}

PALABRAS CLAVES. Arrecife de coral, corales negros, comunidades coralinas, buceo autónomo, conservación

\section{ASTRACT}

The marine environments in the continental shelf within the influence zone of Coiba National Park have important ecological attributes that maintain a close relationship with this protected area. Due to this particular trait, a characterization of marine bottoms in Ensenada de Pixvae (comanagement area for responsible fishing), was conducted by describing and documenting the main ecological aspects by means of photography and substrate sketches to represent the ecological features and its associated marine species diversity. The survey indicated opportunity to develop sustainable activities independent from fishing such as, scientific and recreational diving, which can provide economical alternatives for the local communities, with a focus on recovery and conservation of the system. There is no doubt; the characteristics of coralline communities associated to the bottoms of Ensenada de Pixvae possess attributes that generate interest for development of ecological tourism.

KEYWORDS. Coral reef, black coral, coralline communities, autonomous diving, conservation

Artículo recibido: 25 de agosto de 2020

Artículo aceptado: 28 de septiembre de 2020

\section{INTRODUCCIÓN}

A partir de los descubrimientos realizados por Peter Glynn, en la década de los 70, donde documenta la existencia de arrecifes coralinos en el Pacífico de Panamá, se abre la ventana a un 
mundo de investigaciones marinas en tema de arrecifes y comunidades coralinas en la región. Para este muevo ambiente de estudio se ha generado importante información para establecer líneas de gestión enfocadas en la conservación y uso sustentable de los recursos marinos en áreas marinas de gran interés como es el Parque Nacional Coiba (PNC), uno de los parques naturales con mayor extensión marina de la región (Glynn et al., 1972; Guzmán et al., 2004; ANAM, 2009).

Para el PNC se ha documentado que presenta las mayores coberturas de arrecife de coral en la región, donde se han identificado hasta 52 especies de octocorales, muchas endémicas, que brindan un ambiente que sostienen una alta biodiversidad con complejas interacciones y procesos ecológicos claves en pináculos submarinos, como las agregaciones reproductivas de especies de importancia para la pesca comercial (Guzmán \& Breedy, 2008; Vega et al, 2016). Sin embargo, durante mucho tiempo, el aprovechamiento de recursos, tanto en el PNC como en su zona de influencia, se ha enfocado principalmente en la extracción de recursos pesqueros (Montenegro, 2007), y desde la promulgación del plan de manejo del PNC, en el 2009, su implementación ha sido muy baja, lo que ha llevado a una mayor presión sobre los recursos y el medio. Ante esta preocupación, se crea la primera zona de co-manejo en la bahía de Pixvae, zona de amortiguamiento del PNC, mediante resuelto ADM-ARAP-2019, gracias al interés de la comunidad y principalmente al apoyo de actores claves durante el proceso, como la académica, las ONGs, la comunidad y las autoridades. Es así como este documento describe, de manera general, los principales atractivos para el buceo recreativo y científico, dentro del área de co-manejo, con miras a potencializar actividades que brinden beneficios económicos más allá de la pesca, con una base científico-técnica que permita dar seguimiento al impacto producido por las actividades de buceo y pesca que se puedan desarrollar en el sitio.

\section{MATERIALES Y METODOS}

Área de estudio: La zona de co-manejo para la pesca responsable en la bahía de Pixvae, cuenta con un área marina que cubre una extensión superficial de $34.11 \mathrm{~km}^{2}$, una longitud de norte a sur de 2.3 millas náuticas, un ancho máximo de 4.6 millas náuticas y se encuentra entre la bahía de Punta Muerto al norte $\left(81^{\circ} 36^{\prime} 16.33^{\prime} ' \mathrm{~W}-7^{\circ} 52^{\prime} 48.1^{\prime \prime} \mathrm{N}\right)$ a Punta Gorda $\left(81^{\circ} 35^{\prime} 36.27^{\prime}\right.$ ' W $7^{\circ} 48^{\prime} 13.60^{\prime \prime} \mathrm{N}$ ). Su litoral se conforma de playas de arena y roca, sustratos fangosos asociados parches de manglar y la descarga de afluentes como río Muerto, río La Aguja, río Rosario, río Pixvae, río Seco y río Mona.

Descripción de los sitios: Con el apoyo de pescadores locales se realizaron recorridos en la costa de Pixvae para identificar los principales bajos (promontorios submarinos) utilizados para la pesca y extracción de invertebrados. Se consideraron sitios con profundidades dentro de los límites de buceo recreativo ( $<30 \mathrm{~m}$ de profundidad), se sondearon mediante ecosonda y una vez posicionado sobre el bajo, estos fueron georreferenciados. La descripción de la vida marina en cada sitio se hizo con base a componentes ecológicos (riqueza, abundancia, diversidad) para la composición de los bentos, según protocolo establecido en la metodología de Garza-Pérez (2011), modificado de Aronson y Swanson (1997). De igual manera, se realizaron censos visuales para describir el elenco de peces conspicuos y buceos en los extremos de cada bajo para una mejor descripción de los sitios (Montes et al., en prensa). Con esta información y las observaciones realizadas en cada sitio, se procedió a realizar una serie de representaciones esquemáticas que apoyan la descripción de los diferentes sitios. 


\section{RESULTADOS}

La costa de Pixvae cuenta con diversos ambientes submarinos que incluyen desde arrecifes y bajos rocosos, arrecife de coral y comunidades coralinas, fondos de corales negros y fondos arenosos. La descripción y composición de los fondos, por caladero de pesca es la siguiente:

\section{Punta Pixvae}

Bajo La Boya (7'49'41.46''N-81 ${ }^{\circ} 35$ '22.09' 'W): Consiste en una piedra que sube del fondo desde una profundidad de $19 \mathrm{~m}$ en su parte este y $12 \mathrm{~m}$ en su lado oeste, en mareas bajas esta estructura rocosa emerge casi un metro sobre la superficie. Esta formación se dispone de norte a sur, en este último lado pasa un canal de arena a unos $14 \mathrm{~m}$ de profundidad, seguidos de otra formación rocosa que se aproxima hasta unos $8 \mathrm{~m}$ de la superficie, sus paredes rocosas están dominadas por octocorales y algunas esponjas incrustantes.

En su lado oeste, desciende hasta unos $28 \mathrm{~m}$, donde se pueden observar algunas estaciones de limpieza donde participan peces de la familia Labridae y Chaetodontidae, también cuenta con formaciones de corales negros dispersos que cubren las rocas. A unos $5 \mathrm{~m}$ de la superficie, el bajo presenta una porción plana, donde se observa la mayor proporción de corales duros dominado por Pocillopora, y sobre las paredes de la roca gran cantidad de octocorales, de igual manera se aprecian grietas habitadas por peces (Holocentridae, Muraenidae, Serranidae, entre otros) y equinodermos. Por ser un sitio utilizado por pescadores, es común encontrar restos o artes de pesca abandonados como trasmallos, líneas de mano y anzuelos. No se observaron depredadores topes en este sitio, solo un ejemplar juvenil del tiburón punta blanca (Triaenodon obesus).

La estación presenta un porcentaje de cobertura de roca superior al 60\%; a pesar de contar con poca cobertura de corales duros, los mayores arreglos coloniales presentes corresponden a especies como Porites lobata, Pavona clavus y P. gigantea. Por otra parte, en su sector oeste (parte más profunda) se encuentran rocas cubiertas de octocorales (Gorgonidae y Plexauridae) de colores llamativos e importantes coberturas de coral negro Myriopathes panamensis (Figuras 1 y 3 ).

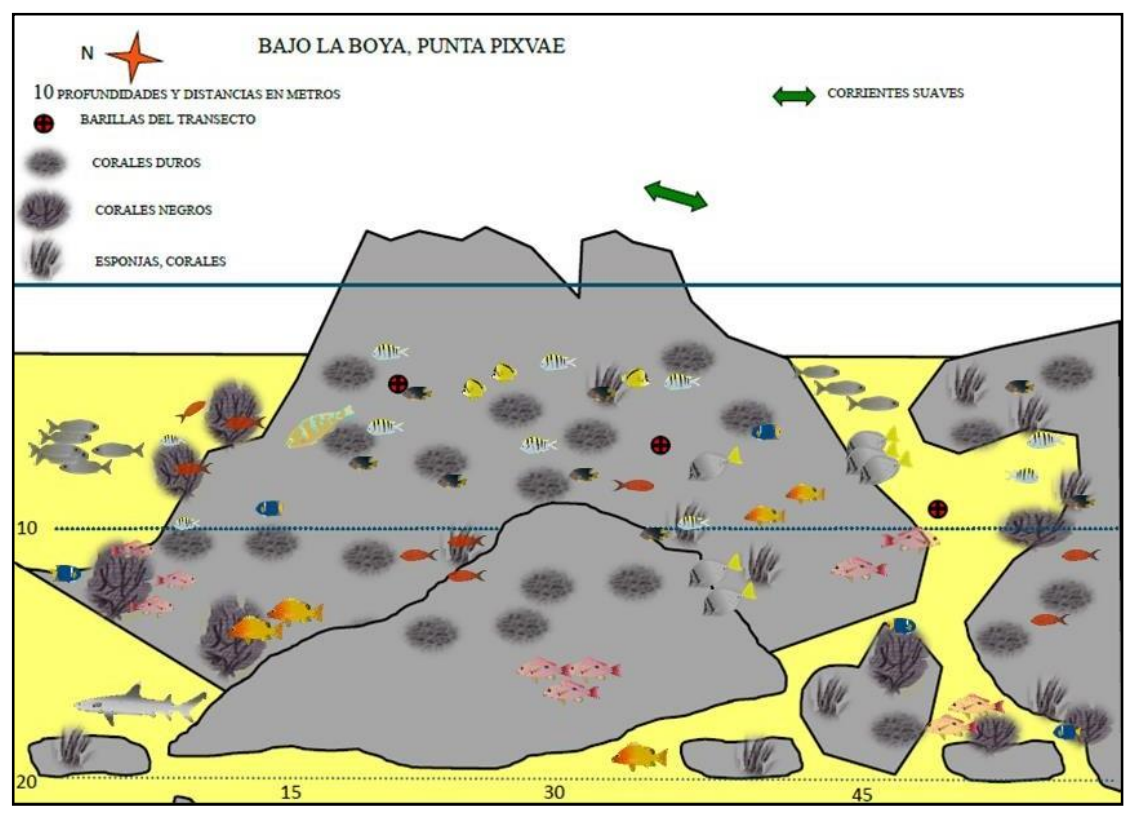

Figura 1. Representación esquemática de Bajo la Boya 
Arrecife de Punta Pixvae (7049'57.5'N-81'35'14.9''W): Localizado en litoral rocoso de Punta Pixvae, el sitio consiste en un arrecife de corales masivos de la familia Agariciidae, compuesto principalmente por Pavona clavus, cuenta con estructuras erectas que alcanzan hasta $1.5 \mathrm{~m}$ de alto, ubicado en un rango de profundidad entre 5 a $8 \mathrm{~m}$; en los bordes de la parte más somera del arrecife se encuentran colonias de coral ramificado (Pocillopora elegans y Pocillopora damicornis) y en menores proporciones Pavona varians. La estructura del arrecife permite el asentamiento de una variada vida marina desde organismos bentónicos (equinodermos, tunicados, ascidias, esponjas y octocorales) que aprovechan la disposición de espacios entre el entramado de canales y agujeros que forma el arrecife (Figuras 2 y 3 ).

Caso similar sucede con el elenco de peces conspicuos, se aprecian importantes densidades de peces coralinos de las familias Pomacentridae, Haemulidae, Lutjanidae y Holocentridae. Entre el grupo de los pargos se encuentran el pargo amarillo (Lutjanus argentiventris), pargo mancha ( $L$. guttatus) y pargo negro (L. novemfasciatus) (Figura 3).

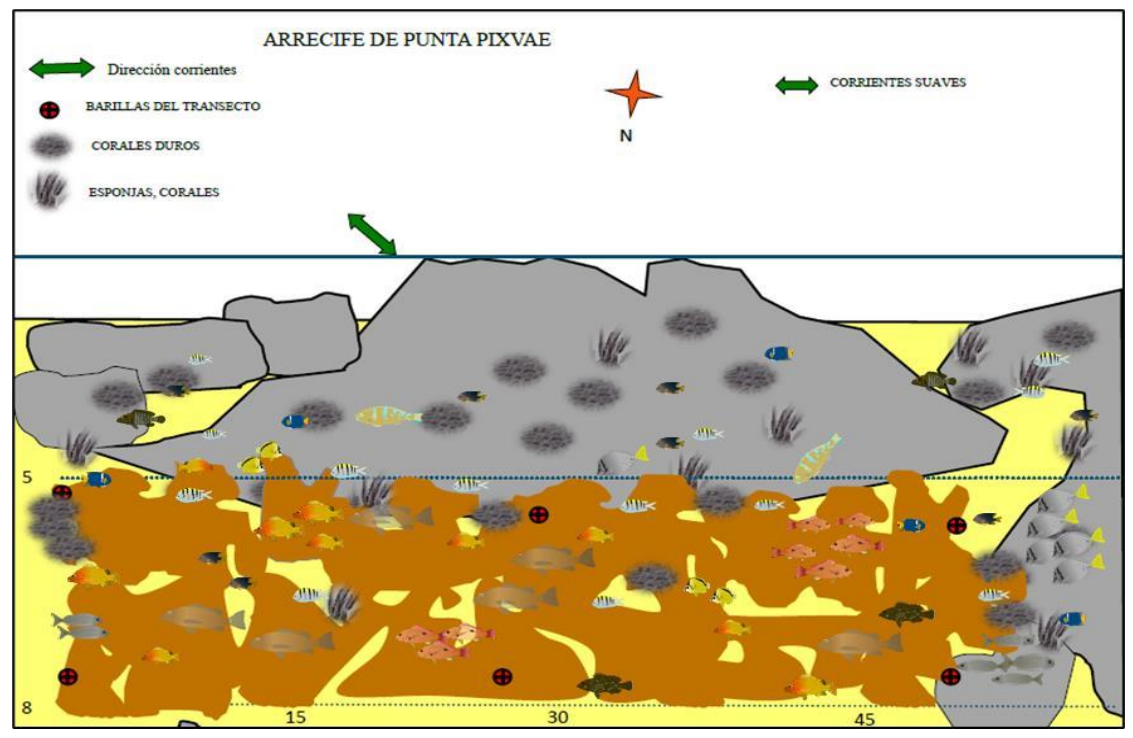

Figura 2. Representación esquemática del Arrecife de Punta Pixvae.

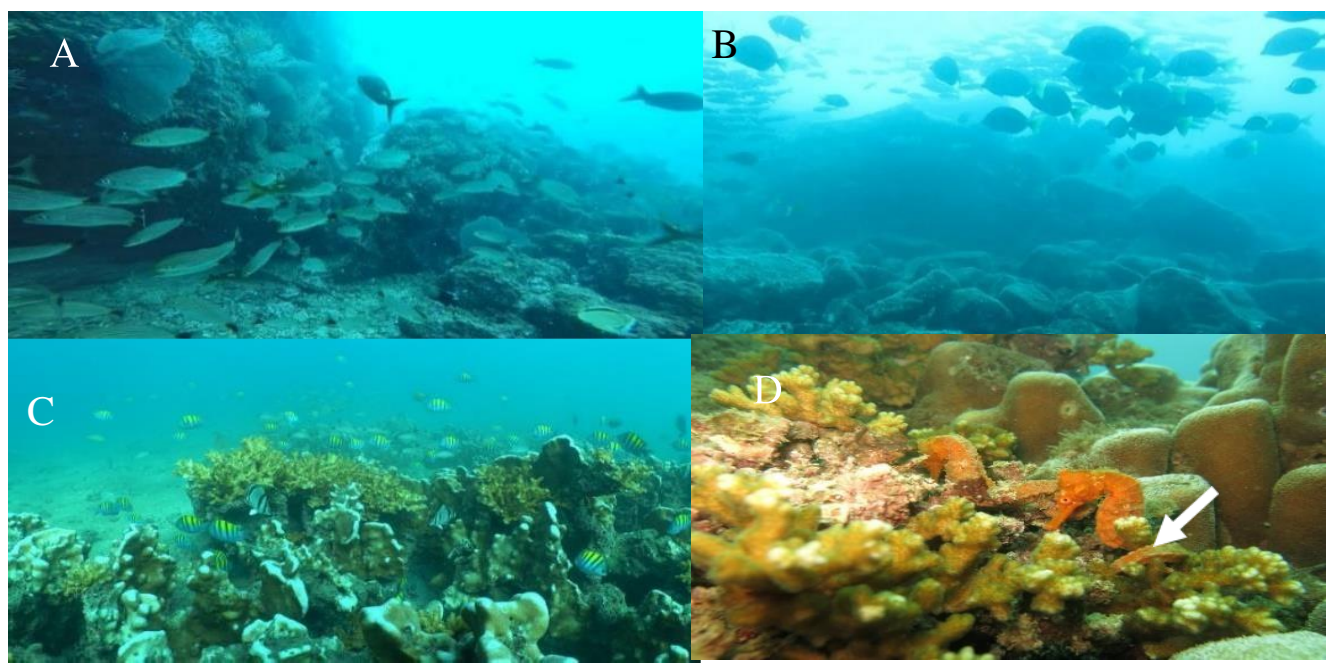

Figura 3. Ambientes marinos de punta Pixvae. A y B) Comunidades coralinas de bajo la Boya; C) Arrecife de punta Pixvae; D) Caballitos de mar (Hippocampus ingens (flecha), aferrados a ramas del coral Pocillopora damicornis en el arrecife de punta Pixvae 


\section{Isla Muela}

Ubicada en la entrada de la ensenada de Pixvae, consiste en sistema de piedras que conforma un arrecife rocoso con comunidades coralinas dispersas, cuenta con características particulares que se aprecian en sentido este de la Isla (hacia el continente), con el lado oeste (hacia el océano). Estos ambientes cuentan con una variada ictiofauna dominado por peces de arrecife, también, es común el avistamiento de peces de hábitos pelágicos y tortugas marinas, que ingresan en jornadas de alimentación o por las estaciones de limpieza.

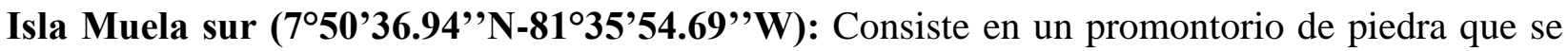
eleva de 6 a $8 \mathrm{~m}$ en su parte más cercana a la superficie, está relativamente plana (su parte somera) y abarca un área próxima a los $30 \mathrm{~m}^{2}$, se observan grandes colonias de corales masivos como Pavona clavus y Porites lobata, de igual forma, parches de Pocillopora (P. elegans, P. damicornis y $P$. eydouxi); en sus parte más profunda desde los 15 a \pm 20 m donde termina la roca, se encuentran octocorales de los géneros Pacifigorgia y Leptogorgia.

El elenco de peces conspicuos lo conforman peces coralinos (Pomacentridae, Labridae, Scaridae, Haemulidae y Serranidae). Este sito, al tener influencia oceánica, es propenso al ingreso de grupos de carángidos (Caranx caballus y $C$. sexfasciatus), como también importantes avances de sardinas (durante la temporada seca) que atraen peces cazadores de nado activo. Por otra parte, donde termina la roca no se encontró encontrames con un fondo de arena que conecta con otros bajos, en este fondo arenoso se encuentra otro tipo de vida donde se observan rayas (Dasyatidae y Rhinobatidae), serránidos (Diplectrum sp.), lenguados (Achiridae, Cynoglossidae) y peces escorpión (Scorpaenidae), además de anemonas y estrellas de mar (Figura 4).

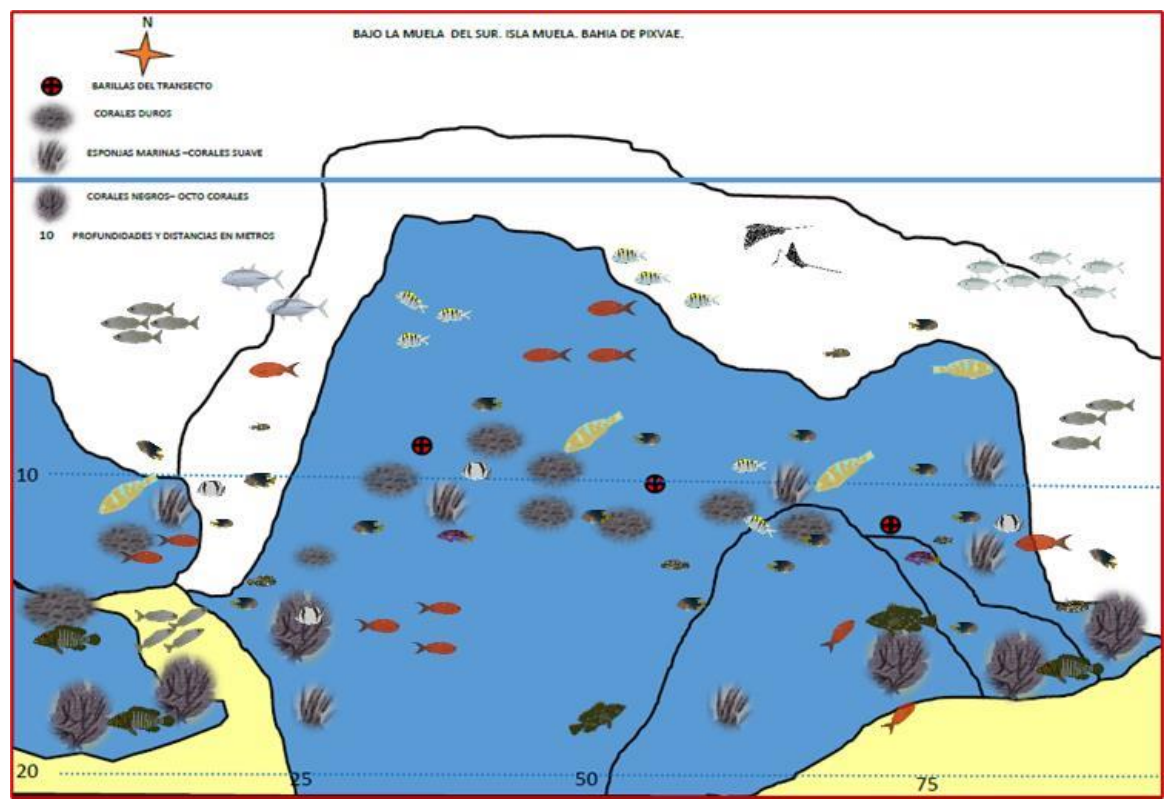

Figura 4. Representación esquemática del bajo de Isla Muela Sur 
Isla Muela Oeste $\left(7^{\circ} 50{ }^{\prime} 38.63 '\right.$ 'N-81 35 '55.87' $\left.\mathbf{W}\right)$ : Un bajo muy similar, en lo que atributos físicos y ecológicos se refiere, con sector sur. Cerca de los $20 \mathrm{~m}$ se encuentra la roca con la arena, pero cuenta con una caída en sentido oeste que llega hasta $\pm 60 \mathrm{~m}$; esta parte de la isla tiene mayor influencia de corrientes marinas y oleaje, pero presenta poca cobertura de coral, las especies presentes consisten en arreglos coralinos del género Pavona y colonias ramificados de Pocillopora, de manera dispersa. Se ha observado agregaciones de Pentaceraster cumigi de más de 70 individuos, además del erizo Toxopneustes roseus. En tiempo de avistamiento de ballenas jorobadas (Megaptera novaeangliae), se les observa bien cerca del bajo.

Isla Muela Este $\left(7^{\circ} 50^{\prime} 41.57^{\prime}\right.$ 'N-81 35 '51.82'’ W): Se posiciona frente a la costa de Pixvae, cuenta con una profundidad de $\pm 10 \mathrm{~m}$ donde la roca se encuentra con la arena y llega hasta los $15 \mathrm{~m}$ a medida que se nada en dirección norte de la isla, donde se ubican rocas de gran tamaño ( $>3 \mathrm{~m}) \mathrm{y}$ que son independientes de la isla. Por estar relativamente protegida de la influencia oceánica, en este sito se observan las mayores coberturas de coral escleractino con respecto a las demás comunidades coralinas de Pixvae, dominada por colonias de corales ramificados pocilopóridos (Pocillopora elegans, $P$. damicornis y P. eydouxi) y del coral masivo Porites lobata, que se asientan sobre el sustrato rocoso compuesto por rocas de tamaños variados (Figura 5).

La estructura de la comunidad de peces conspicuos estuvo bajo el dominio de peces de arrecife, se reportan cerca de 22 especies, también es un sector donde confluyen peces como la salema (Kyphosus oscyurus), roncadores (Haemulon maculicauda), pargo chotillo (Lutjanus colorado), además de tortugas marinas (Chelonia mydas agassizii). Estos ambientes alrededores de isla Muela, cuenta con gran presencia de equinodermos como erizos, pepinos y estrellas de mar, se encuentran cerca de 4 especies de estrellas marinas, con una presencia importante de la estrella corona de espinas (Acanthaster planci) alimentándose tanto de colonia de corales masivos, ramificados e incluso corales negros. Un punto de importancia es la presencia de pargo coliamarillo (Lutjanus argentiventris), que convierte al sitio en un punto de interés pesquero, lo que se pone de manifiesto por los restos de redes de enmalle (trasmallos), cuerdas y plomos, que se pueden encontrar en el bajo.

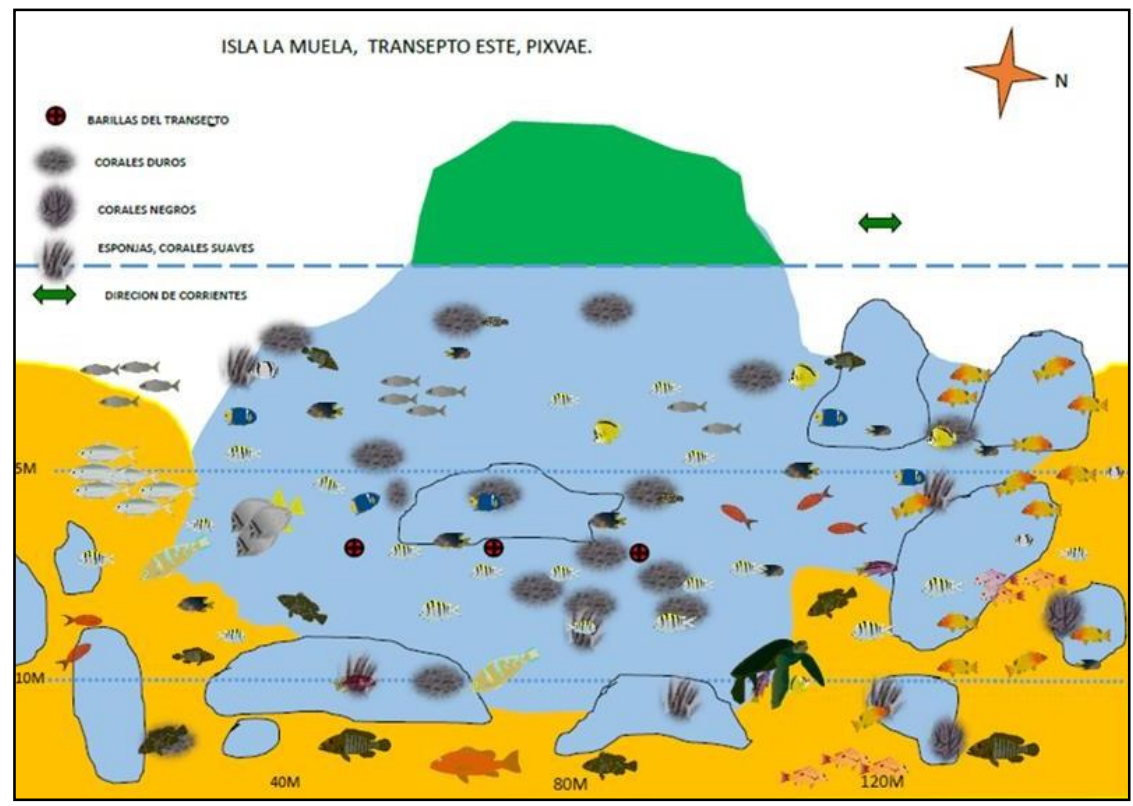

Figura 5. Representación esquemática de Bajo Muela Este 


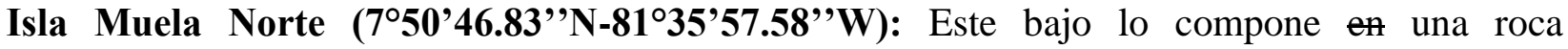
independiente de la isla, se sitúa a una distancia de aproximadamente $150 \mathrm{~m}$ en dirección norte, su parte más somera alcanza entre 6-8 metros, cerca de la superficie en donde se asientan las principales colonias de coral escleractinio, cuenta con gran influencia de corrientes lo que condiciona la presencia de octocorales y esponjas erectas bien desarrolladas (hasta de $0.50 \mathrm{~m}$ ). Este es un bajo amplio que, incluso en su lado este, se puede conectar con la parte oeste de Isla Muela por medio de un canal de arena de entre 15-20 m de profundidad, mientras su sector sur presenta una caída de más de $60 \mathrm{~m}$. Este bajo es un punto con presencia importante de equinodermos, hasta 5 especies de erizos (Diadema mexicanum, Toxopneustes roseus, Astropyga pulminata, Eucidaris thouarsii y Tripneustes depressus), estrellas de mar y pepinos (Figura 6 y 7).

Durante la evaluación, se pudo observar solo un ejemplar de tiburón punta blanca (Triaenodon obesus) en cuevas formadas donde se encuentra la roca con la arena; por otra parte, la estructura de peces estuvo compuesta de peces coralinos, en menor diversidad que los ambientes de isla Muela, sin embargo, en este sitio se puede observar cardúmenes de peces nadadores como barracudas (Sphyraenidae), catecismos (Ephipiidae) y cojinúa (Caranx caballus), además de rayas águila (Aetobatus narinari) y rayas bentónicas.

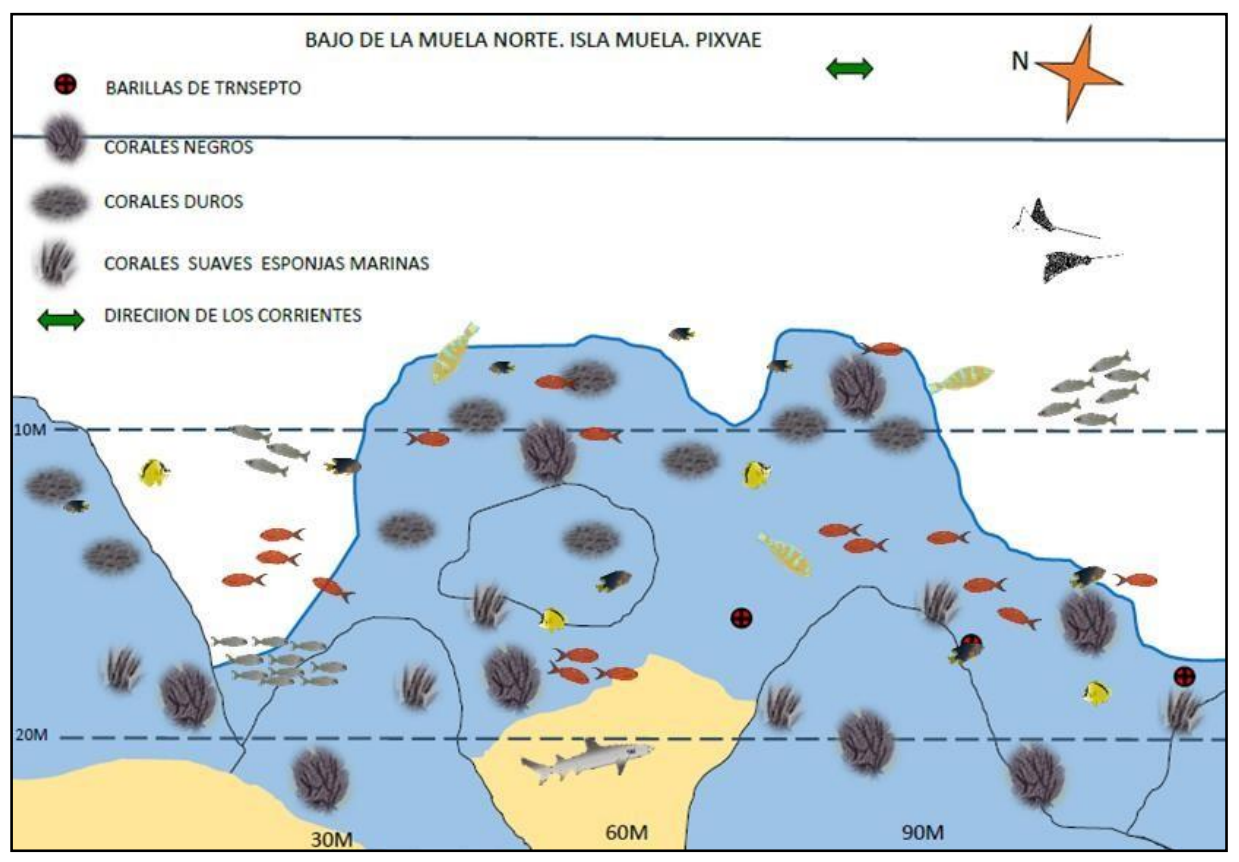

Figura. 6. Representación esquemática de Bajo Muela Norte 

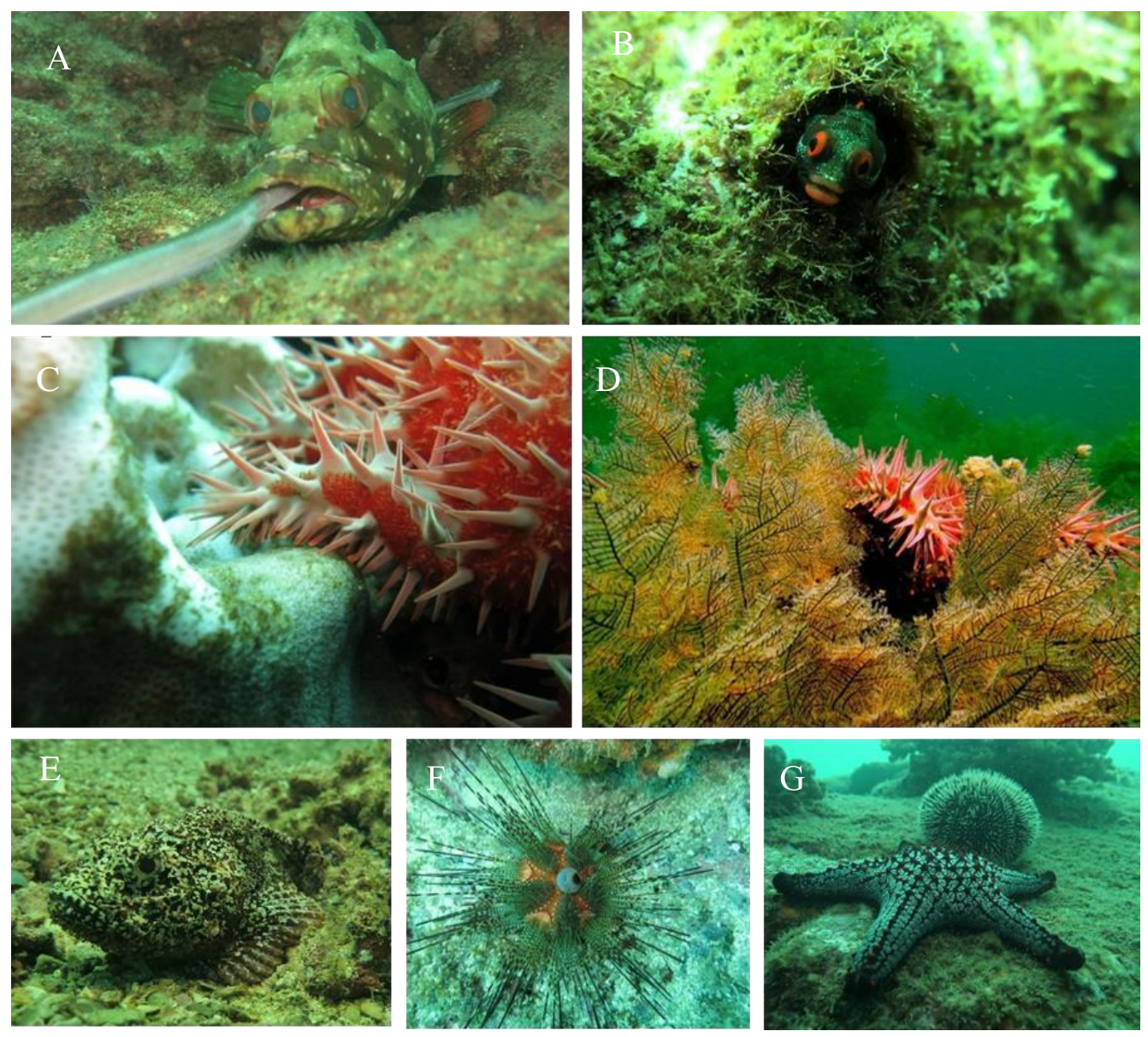

Fig. 7. Fauna asociada a Isla Muela. A) La cabrilla de piedra (Epinephelus labriformis), alimentándose de un pez corneta (Fistulariidae); B) Pez Trambollin (Acanthemblemaria hancocki), habita en tubos vacíos de cirrípedos, poliquetos o moluscos en arrecifes de coral y rocosos; C y D) Estrella corona de espinas (Acanthaster planci) alimentándose de colonias de coral duro y negro; E) Pez escorpión, se mimetiza en los fondos de arena entre Isla Muela y el Bajo de Isla Muela Norte; F) El erizo (Astropyga pulminata) en Isla Muela Norte; G) Equinodermos en el bajo Norte de isla Muela.

\section{Bahía de Pixvae}

Cuenta con una serie de bajos de roca volcánica y sedimentaria, que se disponen en línea recta en dirección oeste a partir de Punta Moliné; por su cercanía a la costa, se encuentra influenciados por la escorrentía proveniente de tierra firme, además del aporte de sedimentos por parte de los ríos Pixvae, Seco y Mona. A pesar de ello, es diverso en corales duros, 10 especies, y peces coralinos con 25 especies, principalmente en bajos someros que emergen durante la baja mar.

Bajo Ruth (7'50'18.32',-N81 ${ }^{\circ} 35$ '5.85' $\left.' W\right)$ : El sitio cubre aproximadamente $110 \mathrm{~m}$ de largo por $40 \mathrm{~m}$ de ancho, orientado de oeste a este, en su parte más somera alcanza $8 \mathrm{~m}$ cerca de la superficie, el fondo mayormente rocoso $\pm 85 \%$ cuenta con canales de arena y una profundidad cerca de $14 \mathrm{~m}$ en el sector este, su parte oeste desciende hasta los $24 \mathrm{~m}$; un aspecto importante, es que gran parte de la superficie tanto roca como coral se encuentra influenciada por el aporte de sedimentos. La 
complejidad de la roca ofrece hábitat apropiado para el asentamiento de organismo bentónicos (erizos, estrellas, pepinos, peces sapo y langosta), además se observan arreglos de octocorales y esponjas en los alrededores de la roca (Figura 8).

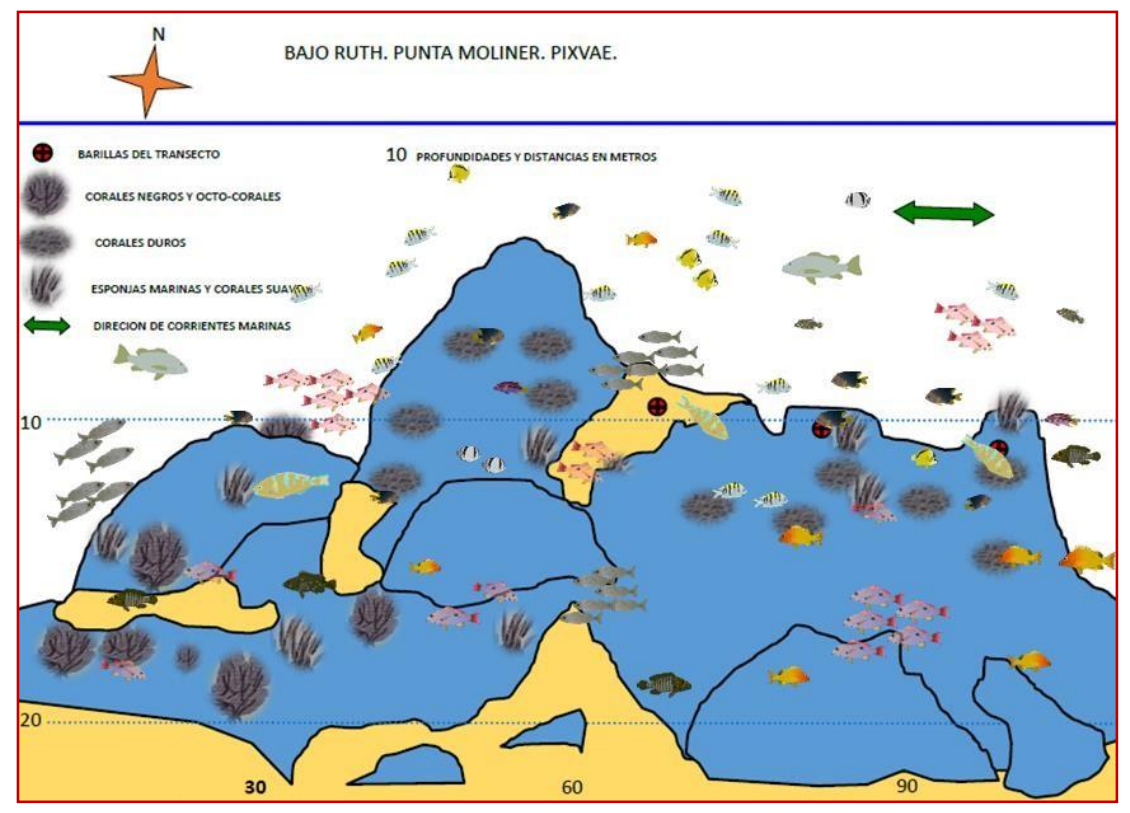

Figura 8. Representación esquemática de Bajo Ruth

El sitio cuenta con una amplia representación de peces de arrecife, también se pueden encontrar varias especies de pargos como el coliamarillo, pargo mancha, este último en grandes densidades principalmente en verano; asociados a movimiento de masas de agua fría, otras especies como el pargo negro (Lutjanus novemfasciatus); se observan algunos individuos en etapa de subadultos y rabirrubia se puede encontrar en grandes agrupaciones (Lutjanus inermis).

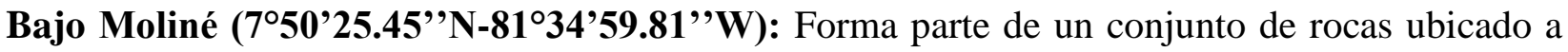
$140 \mathrm{~m}$ de punta Moliné, algunas de estas emergen durante la baja mar, por lo que mantienen una dinámica constante por la rompiente; el bajo está a unos 6 metros de la superficie en su parte más somera, está constituido por roca en un $60 \%$, dividido por varios canales de arena. El sitio cuenta con formaciones de corales masivos principalmente Porites lobata y Pavona gigantea, donde se resguardan peces de interés comercial; además presenta diferentes arreglos de esponjas y organismos asociados. En los pargos se identificaron cinco especies (en etapas juveniles), al menos 4 especies de morenas, peces loro y un elenco de peces coralinos dominados por las familias Pomacentridae y Labridae. Los organismos en este sitio muestran complejas interacciones, se cuenta con estaciones de limpieza que aprovechan las tortugas marinas (Erectmochelis imbricata), también se observan peces depredadoras que se mimetizan con la roca como sucede con los peces escorpiones, o con arreglos de esponjas erectas como hace el pez sapo, además se han observado babosas marinas o nudibranquios de arreglos y colores exuberantes. Por otra parte, la influencia de la costa por la descarga de los ríos Mona y Pixvae, y la escorrentía superficial, durante lluvias fuertes enturbia toda la bahía, causando estrés en las colonias de corales escleractinios, debido a que llegan a estar completamente cubierta de algas y sedimentos. A pesar de estas limitantes, es un ambiente diverso tanto de organismo sésiles como también de peces (Figuras 9 y 10). 


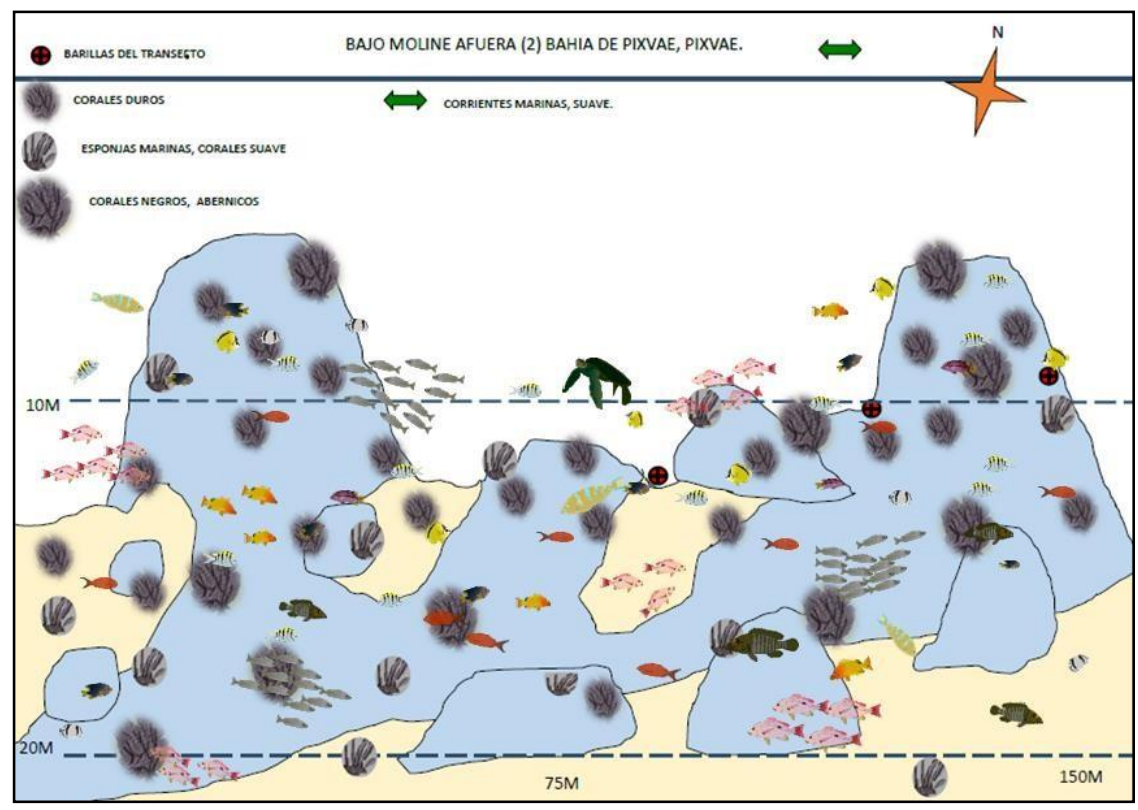

Figura. 9. Representación esquemática de Bajo Moliné

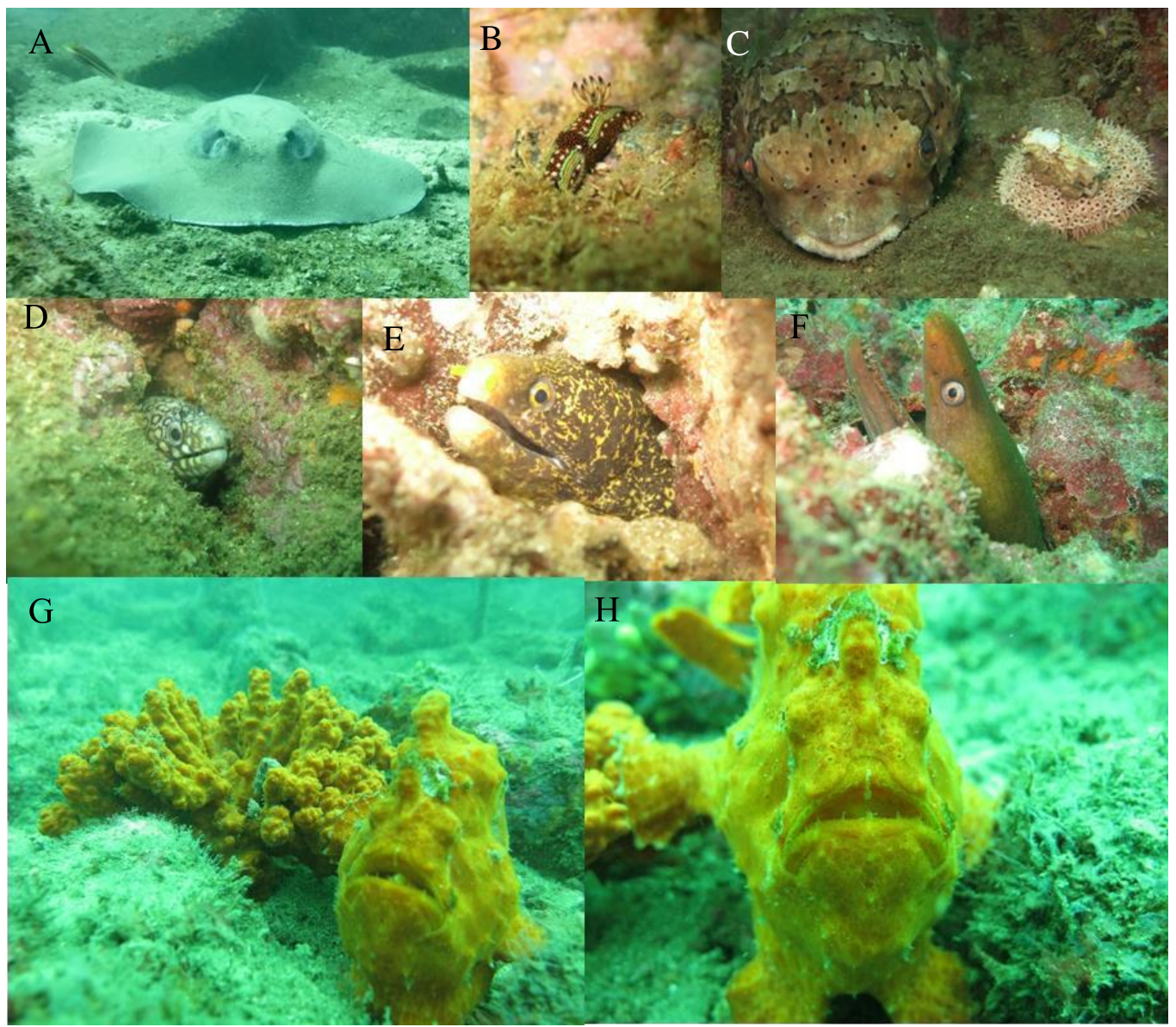

Figura 10. Organismos en los bajos de la bahía de Pixvae. A) Raya latigo (Dasyatis longus); B) Nudibranquio (Hypselodoris agassizzi); C) Izquierda: Pez erizo (Diodon holacanthus), Derecha: erizo (Toxopneustes roseus); D) Morena joya (Muraena lentiginosa); E) Morena estrellada (Echidna nebulosa); G) Gymnothorax castaneus; H) Esponja (Axinella sp.) lado izquierdo; H) Pez sapo (Antennarius commerson) 


\section{Bahía de Rosario}

Al norte de la bahía de Pixvae, entre Punta Doña Juana y Punta Muerto se encuentra la bahía de Rosario, cuenta con un litoral rocoso, playas de arena, piedra y fango, además de pequeños parches de manglar asociado a la desembocadura del río Rosario, este al igual que río La Aguja descargan en la bahía. Esta bahía es de gran interés para la captura de pargo seda (Lutjanus peru) y pargo mancha (Lutjanus guttatus), pescadores locales esperan la entrada de grandes densidades de juveniles durante los primeros meses del año, que ingresan al sistema asociados a movimiento de agua fría; de igual manera, cuenta con sitos de interés para el buceo, que comprenden fondos arenosos, cascajo, pináculos rocosos y ambientes de corales negros.

Bajo Goyo (751'40.01N-81'35'1.22''W): Ubicado en el centro de la bahía, este bajo consiste en un promontorio de piedra, los ángulos del estrato de esta cordillera rocosa que sale de la arena están orientados de norte a sur, con los puntos más altos en sentido sur. La parte más somera del sitio se encuentra a $\pm 19 \mathrm{~m}$ con la baja mar, comprende un área aproximada de $80 \mathrm{~m}$ de largo por $30 \mathrm{~m}$ de ancho, por los lados norte-oeste desciende hasta unos 30m, mientras que para el sureste llega hasta los $27 \mathrm{~m}$ de profundidad; la parte rocosa que emerge de la arena se encuentra cubierta de grandes arreglos de corales negros mayores a $1 \mathrm{~m}$ de altura, octocorales y esponjas, en estos escenarios cargados de vida confluyen importantes concentraciones de juveniles de roncadores y pargos, con densidades que superan fácilmente los 500 individuos, lo que lo hace susceptible a la pesca (Fig. 11). Es uno de los pocos sitios donde se ven depredadores topes como mero de profundidad del Pacífico (Epinephelus quinquefasciatus) y pargos negros (Lutjanus novemfasciatus) en etapa adulta; es sin duda un sitio de importancia en el ciclo de vida de especies comerciales como el pargo, sin embargo, se pueden observar restos de equipos de pesca en todo el bajo (Figuras $11 \mathrm{y}$ 12).

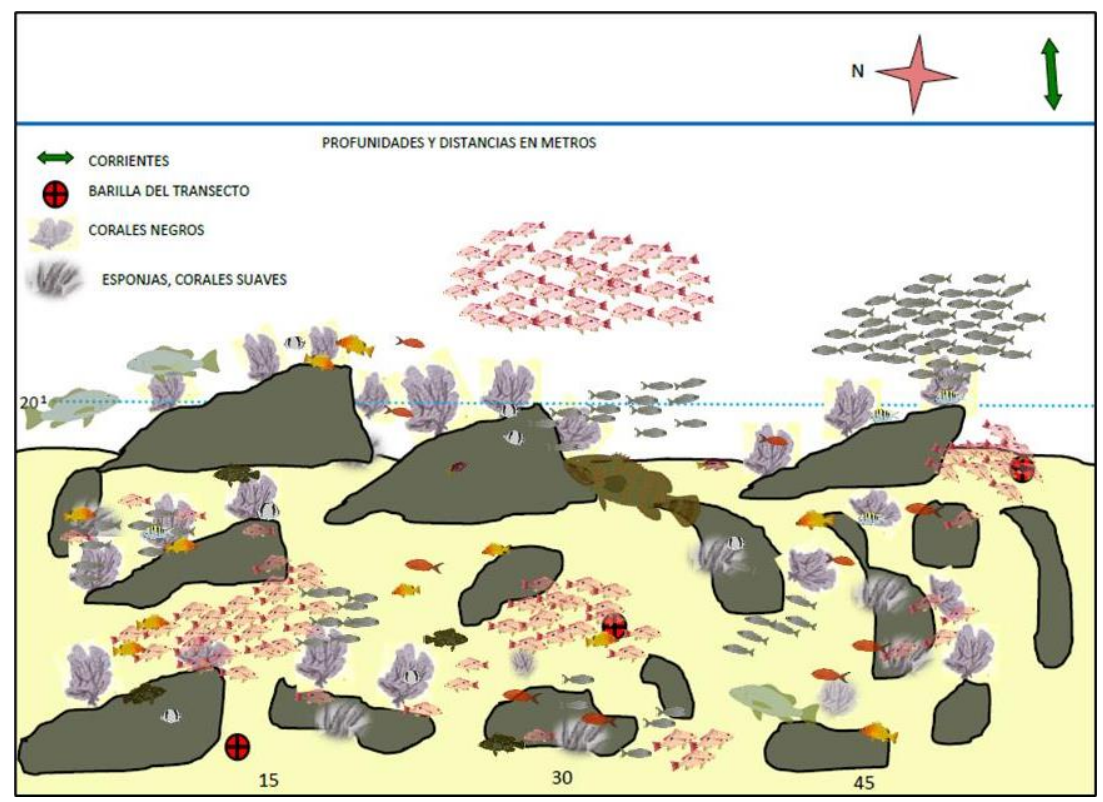

Figura 11. Representación gráfica de Bajo Goyo - Bahía de Rosario, Pixvae 

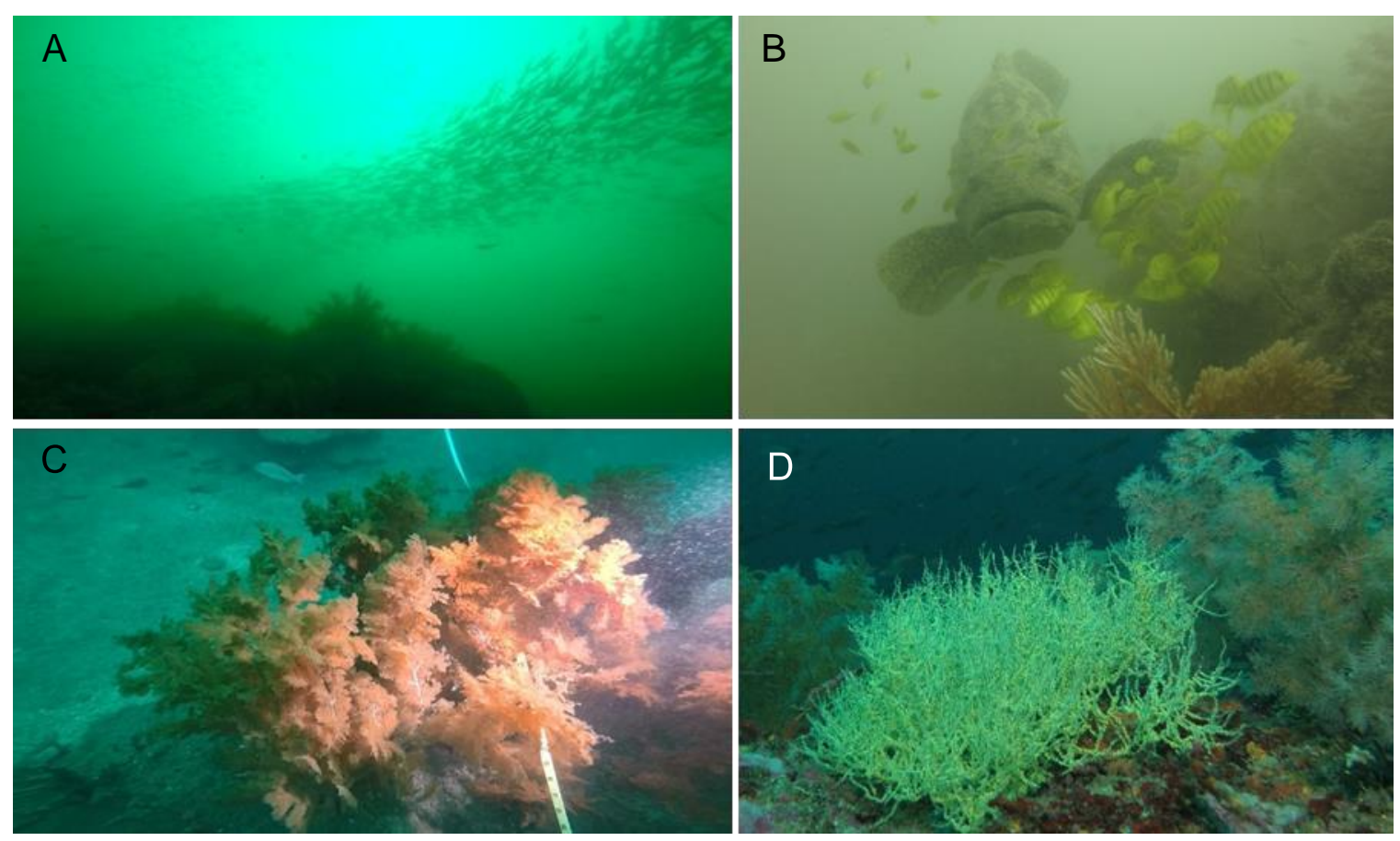

Figura 12. Ambiente de Bajo Goyo. A) Cardumen de pargo mancha (Lutjanus guttatus) sobre el bajo; B) Mero de profundidad del Pacifico (Epinephelus quinquefasciatus); C) Coral negro (Myriopathes panamensis) dominante; D) Coral negro (Antipathes galapagensis).

\section{DISCUSIÓN}

El buceo es una actividad en desarrollo que presenta un crecimiento anual de $20 \%$, en caso del PNC esta es una de las actividades que genera mayores visitas, lo que se traduce en un importante derrame económico para las comunidades costeras en su zona de influencia, principalmente Santa Catalina, que ofrece los servicios básicos necesarios (Barragán \& GalvánVilla, 2016; Mantell \& Maté, 2007), aunque actualmente no se cuenta con un documento que regule la actividad de buceo en el parque y se desconoce la capacidad de carga de los sitios.

A nivel global, se estima que las Áreas Marinas Protegidas (AMP) cubren solo un 3.27\% de los océanos y de estos solo el $0.1 \%$ se encuentra completamente protegido de actividades extractivas, el resto sufre de mala gestión y administración (Boonzaier \& Pauly, 2015). Sin embargo, existen casos exitosos, como parque Cabo Pulmo en México, que mediante decisión comunitaria, se crea un área de no extracción y en un periodo de 20 años, científicos indican que la biomasa de las poblaciones de peces se incrementó en un $400 \%$, resaltando que las áreas marinas protegidas (AMP) administradas localmente y la participación de las partes interesadas, pueden tener más éxito que grandes AMP cargadas en legislación, pero administradas por el gobierno federal (Aburto-Oropeza et al.,2011).

Con la creación de una zona de co-manejo para la pesca responsable en la bahía de Pixvae, se crea un precedente ante la necesidad de conservación y recuperación de sistemas marinos en el área de influencia del PNC. Dominicci-Arosemena \& Wolf (2006), indica que patrones de distribución de peces relacionados con la profundidad muestra que un $71.4 \%$, de las especies presentan tallas relativamente pequeñas en hábitats someros asociados a la franja continental en 
áreas de influencia al Parque, mientras que las especies de mayor tamaño, se asocian a zonas profundas, por lo que ambientes como la ensenada de Pixvae, actúan como guardería para muchas especies de peces; además se han observado nuevas interacciones como las estrellas coronas de espinas (Acanthaster planci) alimentándose de corales negros (Myriopathes panamensis), algo no reportado para el Pacífico panameño. Esto y otros aspectos ofrecen un sinnúmero de posibilidades para realizar ciencia y promover la formación de recurso humano en áreas costeras, con miras al desarrollo comunitario por medio de herramientas como el ecoturismo marino.

Un aspecto importante es que la ensenada de Pixvae es la primera zona de co-manejo declarada para el Pacífico de Panamá por solicitud de la comunidad de pescadores de Pixvae. En este sentido, los aportes de este estudio brindan la oportunidad de tener una línea base que permita dar seguimiento al impacto de actividades como la pesca y el buceo, a través de herramientas simples que miden el cambio producido por estas actividades en los ecosistemas, como es el límite de cambio aceptable (Perera-Valderrama et al., 2007), ya que estudios realizados en Gran Caimán, en el Caribe, demostraron el impacto del buceo sobre áreas sujetas a altos niveles de uso, provocando que la cobertura de coral vivo disminuya de manera proporcional a la intensidad del uso del sitio (Trátalos \& Austin, 2001). Esto implica que el uso de los sitios de buceo en la ensañada de Pixvae debe estar regulado por normas de capacidad de carga y seguimiento de indicadores, que deben estar establecidos en un plan de manejo del área de co-manejo.

\section{CONCLUSIÓN}

Los ambientes marinos de la costa de Pixvae cuentan con atributos naturales, que lo potencian como un sitio de gran interés desde el punto de vista turístico y científico, y que puede redundar en alternativas económicas para la comunidad y que sirvan de modelo de sustentabilidad para el resto de país. Las caracterizaciones presentadas brindan una línea base para dar seguimiento al impacto producido por la visitación y la pesca en los diferentes sitios.

\section{AGRADECIMIENTO}

Se agradece a Conservación Internacional por el apoyo bridando para el desarrollo de este estudio.

\section{REFERENCIAS}

Aburto-Oropeza, O., Erisman, B., Galland, G.R., Mascareñas-Osorio, I., Sala, E. \$ Ezcurra, E. (2011). Large Recovery of Fish Biomass in a No-Take Marine Reserve. PLoS ONE 6(8): e23601.

ANAM. 2009. Plan de Manejo del Parque Nacional Coiba. Compiladores: J.L. Maté, D. Tovar, E. Arcia y Y. Hidalgo. STRI.

Aronson, R.B. \& Swanson, D.W. (1997). Video surveys of coral reefs: uni and multivariate applications. Proc. 8th Int. Coral Reef Sym., 2, 1923-1926.

Barragán-Zepeda, A.K. \& Galván-Villa, C.M. (2016). El Buceo ecológico: una forma de conservar la biodiversidad marina. CONABIO. Biodiversitas, 126:8-11.

Boonzaier, L. \& Pauly, D. (2015). Marine protection targets: an updated assessment of global progress. Fauna \& Flora International. Oryx, 50(1), 27-35. 
Dominici-Arosemena, A. \& Wolff, M. (2006). Reef fish community structure in the Tropical Eastern Pacific (Panamá): living on a relatively stable rocky reef environment. Helgol. Mar. Res 60:287-305.

Garza-Pérez J.R. (2011). Evaluación de comunidades bentónicas arrecifales. Guía de campo y laboratorio. PAPIME, DGAPA-UNAM, 25pp.

Glynn, P.W., Stewart, R.H. \& McCosker, J.E. (1972). Pacific coral reef of Panamá: structures distribution and predators. Geol. Rundschau. 61:483-519.

Guzmán, H., Guevara, CA. \& Breedy, O. (2004). Distribution, diversity, and conservation of coral reefs and coral communities in the largest marine protected area of Pacific Panama (Coiba Island). Environmental Conservation 31 (2): 111-121.

Guzmán, H. \& Breedy, O. (2008). Distribución de la diversidad y estado de conservación de los arrecifes coralinos y comunidades coralinas del Pacífico Occidental de Panamá (Punta Mala, Punta Burica) The Nature Conservancy, 39 p.

Mantell, K. \& Maté, J. 2007. Manual de Buceo para el Parque Nacional de Coiba, en proceso de revisión y publicación, Consultoría para el Plan de Manejo del PN Coiba. ANAM-STRI. Ciudad de Panamá.

Montenegro, R. (2007). Valoración Económica de los Recursos Turísticos y Pesqueros del Parque Nacional Coiba. Conservation Strategy Fund. Panamá, 82 p.

Perera-Valderrama, S., Estrada, R., Hernández-Ávila, A., García-Sáez, J. C., Alcolado-Méndez, P. \& García-Rivas, M. C. (2007). Metodología para la Determinación de la Capacidad de Carga de Visitantes, en las Áreas Marinas Protegidas de Cuba. Ponencia presentada en 58th Gulf and Caribbean Fisheries Institute. Texas, EE. UU.

Trátalos, J. A. \& Austin. T. J. (2001). Impacts of recreational SCUBA diving on coral communities of the Caribbean island of Grand Cayman Biological Conservation. 102: 67-75.

Vega, A.J., Maté, J. \& Robles, Y.A. (2016). First Report of Reproductive Aggregations for Pacific Red Snappers Lutjanus peru (Nicholson y Murphy, 1992) and Spotted Rose Snapper L. guttatus (Steindachner, 1869) in the Coiba National Park, Pacific of Panama. Proceedings of the 68th Gulf and Caribbean Fisheries Institute. (68):112-117. 